\title{
LECTURES ON DERIVED AND TRIANGULATED CATEGORIES
}

\author{
BEHRANG NOOHI
}

These are the notes of three lectures given in the International Workshop on Noncommutative Geometry held in I.P.M., Tehran, Iran, September 11-22.

The first lecture is an introduction to the basic notions of abelian category theory, with a view toward their algebraic geometric incarnations (as categories of modules over rings or sheaves of modules over schemes).

In the second lecture, we motivate the importance of chain complexes and work out some of their basic properties. The emphasis here is on the notion of cone of a chain map, which will consequently lead to the notion of an exact triangle of chain complexes, a generalization of the cohomology long exact sequence. We then discuss the homotopy category and the derived category of an abelian category, and highlight their main properties.

As a way of formalizing the properties of the cone construction, we arrive at the notion of a triangulated category. This is the topic of the third lecture. After presenting the main examples of triangulated categories (i.e., various homotopy/derived categories associated to an abelian category), we discuss the problem of constructing abelian categories from a given triangulated category using $t$-structures.

A word on style. In writing these notes, we have tried to follow a lecture style rather than an article style. This means that, we have tried to be very concise, keeping the explanations to a minimum, but not less (hopefully). The reader may find here and there certain remarks written in small fonts; these are meant to be side notes that can be skipped without affecting the flow of the material. With a few insignificant exceptions, the topics are arranged in linear order.

A word on references. The references given in these lecture notes are mostly suggestions for further reading and are not meant for giving credit or to suggest originality.

Acknowledgement. I would like to thank Masoud Khalkhali, Matilde Marcolli, and Mehrdad Shahshahani for organizing the excellent workshop, and for inviting me to participate and lecture in it. I am indebted to Masoud for his continual help and support. At various stages of preparing these lectures, I have benefited from discussions with Snigdhayan Mahanta. I thank Elisenda Feliu for reading the notes and making useful comments and, especially for creating the beautiful diagrams that I have used in Lecture 2 ! 


\section{Contents}

\section{Lecture 1: Abelian categories}

\section{Lecture 2: Chain complexes}

1. Why chain complexes?

2. Chain complexes

3. Constructions on chain complexes

4. Basic properties of cofiber sequences

5. Derived categories

6. Variations on the theme of derived categories

7. Derived functors

\section{Lecture 3: Triangulated categories}

1. Triangulated categories

2. Cohomological functors

3. Abelian categories inside triangulated categories; $t$-structures $\quad 28$

4. Producing new abelian categories

5. Appendix I: topological triangulated categories 31

6. Appendix II: different illustrations of TR4 31

References 


\section{Lecture 1: Abelian categories}

Overview. In this lecture, we introduce additive and abelian categories, and discuss their most basic properties. We then concentrate on the examples of abelian categories that we are interested in. The most fundamental example is the category of modules over a ring. The next main class of examples consists of various categories of sheaves of modules over a space; a special type of these examples, and a very important one, is the category of quasi-coherent sheaves on a scheme. The idea here is that one can often recover a space from an appropriate category of sheaves on it. For example, we can recover a scheme from the category of quasicoherent sheaves on it. This point of view allows us to think of an abelian category as "a certain category of sheaves on a certain hypothetical space". One might also attempt to extract the "ring of functions" of this hypothetical space. Under some conditions this is possible, but the ring obtained is not unique. This leads to the notion of Morita equivalence of rings. The slogan is that the "ring of functions" on a noncommutative space is well defined only up to Morita equivalence.

\section{Products AND COPRoducts in CATEGories}

References: $\mathrm{HiSt}$, $\mathrm{We}, \mathrm{Fr}, \mathrm{Ma}, \mathrm{GeMa}$.

Let $\mathrm{C}$ be a category and $\left\{X_{i}\right\}_{i \in I}$ a set of objects in C.

The product $\prod_{i \in I} X_{i}$ is an object in C, together with a collection of morphisms $\pi_{i}: \prod_{i \in I} X_{i} \rightarrow X_{i}$, satisfying the following universal property:

$$
\text { Given any collection of morphisms } f_{i}: Y \rightarrow X_{i}, \quad \begin{aligned}
& \prod_{i \in I} X_{i} \\
& \exists ! f>\downarrow^{7} \\
& Y \stackrel{f_{i}}{\longrightarrow} X_{i}
\end{aligned}
$$

The coproduct $\coprod_{i \in I} X_{i}$ is an object in C, together with a collection of morphisms $\iota_{i}: X_{i} \rightarrow \coprod_{i \in I} X_{i}$, satisfying the following universal property:

$$
\begin{aligned}
& \text { Given any collection of morphisms } g_{i}: X_{i} \rightarrow Y, \quad \coprod_{i \in I} X_{i} \\
& \begin{aligned}
\exists ! f^{<} & \uparrow_{\iota_{i}} \\
& X_{i}
\end{aligned}
\end{aligned}
$$

Remark 1.1. Products and coproducts may or may not exist, but if they do they are unique up to canonical isomorphism.

Example 1.2. 
1. $\quad$ = Sets: $\amalg=$ disjoint union, $\Pi=$ cartesian product.

2. $\quad \mathrm{C}=$ Groups: $\amalg=$ free product, $\Pi=$ cartesian product.

3. C = UnitalCommRings: $\coprod_{\text {finite }}=\otimes_{\mathbb{Z}}, \Pi=$ cartesian product.

4. $C=$ Fields: $\amalg=$ does not exist, $\Pi=$ does not exist.

5. $\quad \mathrm{C}=R$-Mod=left $R$-modules, $R$ a ring: $\quad \coprod_{\text {finite }}=\prod_{\text {finite }}=\oplus$, the usual direct sum of modules.

Exercise. Show that in $R$-Mod there is a natural morphism $\coprod_{i \in I} X_{i} \rightarrow \prod_{i \in I} X_{i}$, and give an example where this is not an isomorphism.

\section{Abelian categories}

References: $\mathrm{HiSt}$, $\mathrm{We}, \mathrm{Fr}, \mathrm{Ma}, \mathrm{GeMa}]$.

We discuss three types of categories: Ab-categories, additive categories, and abelian categories. Each type of category has more structure/properties than the previous one.

An $A b$-category is a category $\mathrm{C}$ with the following extra structure: each $\operatorname{Hom}_{\mathrm{C}}(X, Y)$ is endowed with the structure of an abelian group. We require that composition is linear:

$$
\begin{gathered}
X \stackrel{u}{\longrightarrow} Y \stackrel{f}{\longrightarrow} Z \stackrel{v}{\longrightarrow} T \\
u(f+g)=u f+u g, \quad(f+g) v=f v+g v .
\end{gathered}
$$

An additive functor between $A b$-categories is a functor that induces group homomorphisms on Hom-sets.

An additive category is a special type of $A b$-category. More precisely, an additive category is an $A b$-category with the following properties:

- There exists a zero object 0 such that

$$
\forall X, \operatorname{Hom}_{\mathrm{C}}(0, X)=\{0\}=\operatorname{Hom}_{\mathrm{C}}(X, 0) .
$$

- Finite sums exist. (Equivalently, finite products exist; see Proposition 2.1.)

Before defining abelian categories, we discuss some basic facts about $A b$-categories.

Proposition 2.1. Let $\mathrm{C}$ be an Ab-category with a zero object, and let $\left\{X_{i}\right\}_{i \in I}$ be a finite set of objects in $\mathrm{C}$. Then $\amalg X_{i}$ exists if an only if $\prod X_{i}$ exists. In this case, $\amalg X_{i}$ and $\prod X_{i}$ are naturally isomorphic.

Proof. Exercise. (Or See HiSt.) 
Notation. Thanks to Proposition 2.1 it makes sense to use the same symbol $\oplus$ for both product and coproduct (of finitely many objects) in an additive category.

Exercise. In Example 1.2 which ones can be made into additive categories?

A morphism $f: B \rightarrow C$ in an additive category is called a monomorphism if

$$
\forall X \stackrel{g}{\longrightarrow} B \stackrel{f}{\longrightarrow} C, \quad f \circ g=0 \quad \Rightarrow \quad g=0 .
$$

A kernel of a morphism $f: B \rightarrow C$ in an additive category is a morphism $i: A \rightarrow B$ such that $f \circ i=0$, and that for every $g: X \rightarrow B$ with $f \circ g=0$

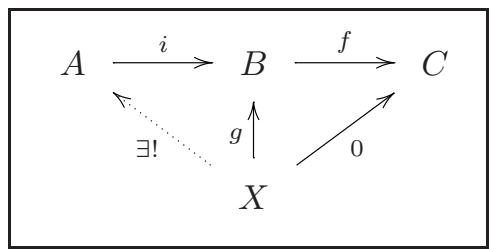

Proposition 2.2. If $i$ is a kernel for some morphism, then $i$ is a monomorphism. The converse is not always true.

We can also make the dual definitions.

A morphism $f: B \rightarrow C$ in an additive category is called an epimorphism if

$$
\forall B \stackrel{f}{\longrightarrow} C \stackrel{h}{\longrightarrow} D, \quad h \circ f=0 \quad \Rightarrow \quad h=0 .
$$

A cokernel of a morphism $f: B \rightarrow C$ in an additive category is a morphism $p: C \rightarrow D$ such that $p \circ f=0$, and such that for every $h: B \rightarrow Y$ with $h \circ f=0$

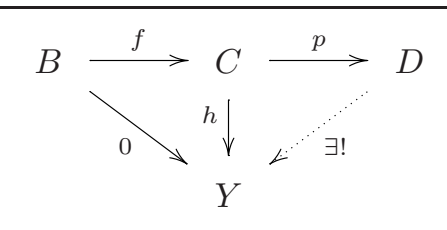

Proposition 2.3. If $p$ is a cokernel for some morphism, then $p$ is an epimorphism. The converse is not always true.

Remark 2.4. Kernels and cokernels may or may not exist, but if they do they are unique up to a canonical isomorphism.

Example 2.5.

1. In $\mathrm{A}=R$-Mod kernels and cokernels always exist. 
2. Let $A$ be the category of finitely generated free $\mathbb{Z}$-modules. Then kernels and cokernels always exist. (Exercise. What is the cokernel of $\mathbb{Z} \stackrel{\times 3}{\longrightarrow} \mathbb{Z}$ ?)

3. Let $\mathrm{A}$ be the category of $\mathbb{C}$-vector spaces of even dimension. Then kernels and cokernels do not always exist in A. (Give an example.) The same thing is true if $A$ is the category of infinite dimensional vector spaces.

An abelian category is an additive category A with the following properties:

- Kernels and cokernels always exist in A.

- Every monomorphism is a kernel and every epimorphism is a cokernel.

Main example. For every ring $R$, the additive category $R$-Mod is abelian.

Remark 2.6. Note that an Ab-category is a category with an extra structure. However, an additive category is just an $A b$-category which satisfies some property (but no additional structure). An abelian category is an additive category which satisfies some more properties.

Exercise. In Example 2.5 show that (1) is abelian, but (2) and (3) are not. In (2) the map $\mathbb{Z} \stackrel{\times 3}{\longrightarrow} \mathbb{Z}$ is an epimorphism that is not the cokernel of any morphism, because it is also a monomorphism!

Proposition 2.7. Let $f: B \rightarrow C$ be a morphism in an abelian category. Let $i: \operatorname{ker}(f) \rightarrow B$ be its kernel and $p: C \rightarrow \operatorname{coker}(f)$ its cokernel. Then there is a natural isomorphism $\operatorname{coker}(i) \stackrel{\sim}{\longrightarrow} \operatorname{ker}(p)$ fitting in the following commutative diagram:

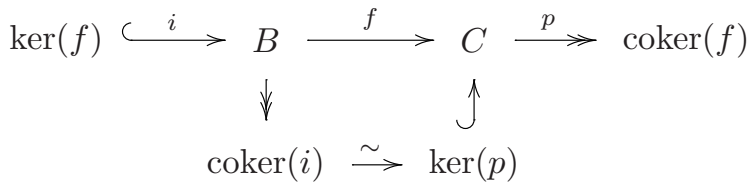

Corollary 2.8. Every morphism $f: B \rightarrow C$ in an abelian category has a unique (up to a unique isomorphism) factorization

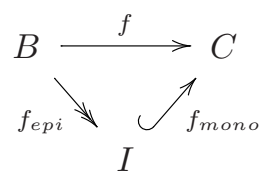

Corollary 2.9. In an abelian category, mono $+\mathrm{epi} \Leftrightarrow$ iso.

The object $I$ (together with the two morphisms $f_{\text {epi }}$ and $f_{\text {mono }}$ ) in the above corollary is called the image of $f$ and is denoted by $\operatorname{im}(f)$. The morphism $f_{\text {mono }}$ factors through every monomorphism into $C$ through which $f$ factors. Dually, $f_{e p i}$ factors through every epimorphism originating from $B$ through which $f$ factors. Either of these properties characterizes the image. 
In an abelian category, a sequence

$$
0 \longrightarrow A \stackrel{f}{\longrightarrow} B \stackrel{g}{\longrightarrow} C \longrightarrow 0
$$

is called exact if $f$ is a monomorphism, $g$ is an epimorphism, and $\operatorname{im}(f)=\operatorname{ker}(g)$. An additive functor between abelian categories is called exact if it takes exact sequences to exact sequences.

We saw that $R$-modules form an abelian category for every $\operatorname{ring} R$. In fact, every small abelian category is contained in some $R$-Mod. (A category is called small if its objects form a set.)

Theorem 2.10 (Freyd-Mitchell Embedding Theorem [Fr, Mi1]). Let A be a small abelian category. Then there exists a unital ring $R$ and an exact fully faithful functor $\mathrm{A} \rightarrow R$-Mod.

\section{Categories of sheaves}

References: Ha1, KaSch, Iv, GeMa.

The main classes of examples of abelian categories are categories of sheaves over spaces. We give a quick review of sheaves and describe the abelian category structure on them.

Let $C$ be an arbitrary category (base) and A another category (values).

A presheaf on $C$ with values in $A$ is a functor $\mathcal{F}: C^{o p} \rightarrow A$. A morphism $f: \mathcal{F} \rightarrow \mathcal{G}$ of presheaves is a natural transformation of functors. The category of presheaves is denoted by $\operatorname{PreSh}(C, A)$.

Typical example. Let $X$ be a topological space, and let $C=$ Open $_{X}$ be the category whose objects are open sets of $X$ and whose morphisms are inclusions. Let $\mathrm{A}=\mathrm{Ab}$, the category of abelian groups. A presheaf $\mathcal{F}$ of abelian groups on $X$ consists of:

$\triangleright$ A collection of abelian groups

$$
\mathcal{F}(U), \quad \forall U \text { open; }
$$

$\triangleright$ "Restriction" homomorphisms

$$
\mathcal{F}(U) \rightarrow \mathcal{F}(V), \quad \forall V \subseteq U .
$$

Restriction homomorphisms should respect triple inclusions $W \subseteq V \subseteq U$ and be equal to the identity for $U \subseteq U$.

\section{Example 3.1.}

1. (Pre)sheaf of continuous functions on a topological space $X$. The assignment

$$
U \mapsto \mathcal{O}_{X}^{c o n t}(U)=\{\text { continuous functions on } U\}
$$


is a presheaf on $X$. The restriction maps are simply restriction of functions. In fact, this is a presheaf of rings because restriction maps are ring homomorphisms.

2. Constant presheaf. Let $A$ be an abelian group. The assignment

$$
U \mapsto A
$$

is a presheaf of groups. The restriction maps are the identity maps.

Variations. There are many variations on these examples. For instance, in (1) one can take the (pre)sheaf of $C^{r}$ functions on a $C^{r}$-manifold, or (pre)sheaf of holomorphic functions on a complex manifold, and so on. These are called structure sheaves. Idea: Structure sheaves encode all the information about the structure in question (e.g, $C^{r}$, analytic, holomorphic, etc.). So, for instance, a complex manifold $X$ can be recovered from its underlying topological space $X^{\text {top }}$ and the sheaf $\mathcal{O}_{X}^{\text {holo }}$. That is, we can think of the pair $\left(X^{\text {top }}, \mathcal{O}_{X}^{\text {holo }}\right)$ as a complex manifold.

Exercise. Formulate the notion of a holomorphic map of complex manifolds purely in terms of the pair $\left(X^{\text {top }}, \mathcal{O}_{X}^{\text {holo }}\right)$.

Proposition 3.2. Let $\mathrm{C}$ be an arbitrary category, and $\mathrm{A}$ an abelian category. Then $\operatorname{PreSh}(\mathrm{C}, \mathrm{A})$ is an abelian category.

The kernel and cokernel of a morphism $f: \mathcal{F} \rightarrow \mathcal{G}$ of presheaves are given by

$$
\begin{aligned}
\operatorname{ker}(f): & U \mapsto \operatorname{ker}\left(\mathcal{F}(U) \stackrel{f_{U}}{\longrightarrow} \mathcal{G}(U)\right) \\
\operatorname{coker}(f): & U \mapsto \operatorname{coker}\left(\mathcal{F}(U) \stackrel{f_{U}}{\longrightarrow} \mathcal{G}(U)\right)
\end{aligned}
$$

A presheaf $\mathcal{F}$, say of abelian groups, rings etc., on $X$ is called a sheaf if for every open $U \subseteq X$ and every open cover $\left\{U_{\alpha}\right\}$ of $U$ the sequence

$$
\begin{array}{r}
\mathcal{F}(U) \stackrel{\text { res }}{\longrightarrow} \prod_{\alpha} \mathcal{F}\left(U_{\alpha}\right) \longrightarrow \prod_{\alpha, \beta} \mathcal{F}\left(U_{\alpha} \cap U_{\beta}\right) \\
\left(f_{\alpha}\right) \mapsto\left(\left.f_{\alpha}\right|_{U_{\alpha} \cap U_{\beta}}-\left.f_{\beta}\right|_{U_{\alpha} \cap U_{\beta}}\right)
\end{array}
$$

is exact.

Example 3.3. Structure sheaves (e.g., Example 3.11) are sheaves! More generally, every vector bundle $E \rightarrow X$ gives rise to a sheaf of vector spaces via the assignment $U \mapsto E(U)$, where $E(U)$ stands for the space of sections of $E$ over $U$. Is a constant presheaf (Example 3.1,2) a sheaf?

For an abelian category $\mathrm{A}$, we denote the full subcategory of $\operatorname{PreSh}(X, \mathrm{~A})$ whose objects are sheaves by $\mathbf{S h}(X, \mathrm{~A})$.

Proposition 3.4. The category $\operatorname{Sh}(X, \mathrm{~A})$ is abelian.

Remark 3.5. 
1. Monomorphisms in $\mathbf{S h}(X, \mathrm{~A})$ are the same as the ones in $\operatorname{PreSh}(X, \mathrm{~A})$, but epimorphisms are different: $f: \mathcal{F} \rightarrow \mathcal{G}$ is an epimorphism if for every open $U$ and every $a \in \mathcal{G}(U)$,

$$
\begin{aligned}
& \exists\left\{U_{\alpha}\right\} \text {, open cover of } U \text {, such that: } \\
& \forall \alpha,\left.a\right|_{U_{\alpha}} \text { is in the image of } f\left(U_{\alpha}\right): \mathcal{F}\left(U_{\alpha}\right) \rightarrow \mathcal{G}\left(U_{\alpha}\right) .
\end{aligned}
$$

2. Kernels in $\mathbf{S h}(X, \mathrm{~A})$ are defined in the same way as kernels in $\operatorname{PreSh}(X, \mathrm{~A})$, but cokernels are defined differently: if $f: \mathcal{F} \rightarrow \mathcal{G}$ is a morphism of sheaves, the cokernel of $f$ is the

$$
\text { sheaf associated to the presheaf } U \mapsto \operatorname{coker}\left(\mathcal{F}(U) \stackrel{f_{U}}{\longrightarrow} \mathcal{G}(U)\right) \text {. }
$$

We remark that there is a general procedure for producing a sheaf $\mathcal{F}^{s h}$ out of a presheaf $\mathcal{F}$. This is called sheafification. The sheaf $\mathcal{F}^{\text {sh }}$ is called the sheaf associated to $\mathcal{F}$ and it comes with a natural morphism of presheaves $i: \mathcal{F} \rightarrow \mathcal{F}^{\text {sh }}$ which is universal among morphisms $\mathcal{F} \rightarrow \mathcal{G}$ to sheaves $\mathcal{G}$. More details on this can be found in Ha1.

\section{Abelian CATEgory of QuAsi-COHEREnt Sheaves on A SCHEme}

References: Ha1, GeMa.

We give a super quick review of schemes. We then look at the category of quasi-coherent sheaves on a scheme.

The affine scheme $\left(\operatorname{Spec} R, \mathcal{O}_{R}\right)$ associated to a commutative unital ring $R$ is a topological space $\operatorname{Spec} R$, the space of prime ideals in $R$, together with a sheaf of rings $\mathcal{O}_{R}$, the structure sheaf, on it. Recall that, in the topology of $\operatorname{Spec} R$ (called the Zariski topology) a closed set is the set $V(I)$ of all prime ideals containing a given ideal $I$. The sheaf $\mathcal{O}_{R}$ is uniquely determined by the fact that, for every $f \in R$, the ring of sections of $\mathcal{O}_{R}$ over the open set $U_{f}:=\operatorname{Spec} R-V(f)$ is the localization $R_{(f)}$, that is, $\mathcal{O}_{R}\left(U_{f}\right)=R_{(f)}$. In particular, $\mathcal{O}_{R}(\operatorname{Spec} R)=R$. (Note: the open sets $U_{f}$ form a basis for the Zariski topology on $\operatorname{Spec} R$.)

A scheme $X$ consists of a pair $\left(X^{Z a r}, \mathcal{O}_{X}\right)$ where $X$ is a topological space and $\mathcal{O}_{X}$ is a sheaf of rings on $X^{Z a r}$. We require that $X$ can be covered by open sets $U$ such that each $\left(U, \mathcal{O}_{U}\right)$ is isomorphic to an affine scheme. (Here, $\mathcal{O}_{U}$ is the restriction of $\mathcal{O}_{X}$ to $U$.)

Remark 4.1. This definition is modeled on Example 3.1.1; in particular, read the paragraph after the example. 
A sheaf of modules over a scheme $X$ is a sheaf $\mathcal{F}$ of abelian groups on $X^{Z a r}$ such that, for every open $U \subseteq X^{Z a r}, \mathcal{F}(U)$ is endowed with an $\mathcal{O}_{X}(U)$-module structure (and restriction maps respect the module structure).

A sheaf of modules $\mathcal{F}$ over $X$ is called quasi-coherent if for every inclusion of the form Spec $S=V \subseteq U=\operatorname{Spec} R$ of open sets in $X^{Z a r}$ we have

$$
\mathcal{F}(V) \cong S \otimes_{R} \mathcal{F}(U)
$$

Proposition 4.2. The category $\mathcal{O}_{X}$-Mod of $\mathcal{O}_{X}$-modules on a scheme $X$ is an abelian category. The full subcategory $\mathbf{Q u a s i - \mathbf { C o h } _ { X }}$ is also an abelian category.

Example 4.3. To an $R$-module $M$ there is associated a quasi-coherent sheaf $\tilde{M}$ on $X=\operatorname{Spec} R$ which is characterized by the property that $\tilde{M}\left(U_{f}\right)=M_{(f)}$, for every $f \in R$. In fact, every quasi-coherent sheaf on $\operatorname{Spec} R$ is of this form. More precisely, we have an equivalence of categories ([Ha1, Corollary II.5.5)

Quasi-Coh $\operatorname{Spec~} R_{2} \cong R$-Mod.

The category Quasi- $\mathbf{C o h}_{X}$ is a natural abelian category associated with a scheme $X$. This allows us to do homological algebra on schemes (e.g., sheaf cohomology). The following reconstruction theorem states that, indeed, Quasi-Coh $X$ captures all the information about $X$.

Proposition 4.4 (Gabriel-Rosenberg Reconstruction Theorem [Ro]). A scheme X can be reconstructed, up to isomorphism, from the abelian category Quasi-Coh . $_{\text {. }}$

More generally, Rosenberg Ro, building on the work of Gabriel, associates to an abelian category $A$ a topological space Spec $A$ together with a sheaf of rings $\mathcal{O}_{A}$ on it. In the case where $A=\mathbf{Q u a s i}-\mathbf{C o h}_{X}$, the pair ( $\left.\operatorname{Spec} A, \mathcal{O}_{A}\right)$ is naturally isomorphic to $\left(X^{Z a r}, \mathcal{O}_{X}\right)$.

The above theorem is a starting point in non-commutative algebraic geometry. It means that one can think of the abelian category Quasi-Coh $\mathbf{C}_{X}$ itself as a space.

\section{Morita EQuivalence of Rings}

References: We, GeMa].

We saw that, by the Gabriel-Rosenberg Reconstruction Theorem, we can regard the abelian category Quasi- $\mathbf{C o h}_{X}$ as being "the same" as the scheme $X$ itself. The Gabriel-Rosenberg Reconstruction Theorem has a more classical precursor.

Theorem 5.1 (Gabriel $\mathrm{Ga}, \mathrm{Fr}]$ ). Let $R$ be a unital ring (not necessarily commutative). Then $R$-Mod has a small projective generator (e.g., $R$ itself), and is closed under arbitrary coproducts. Conversely, let $\mathrm{A}$ be an abelian category with a small projective generator $P$ which is closed under arbitrary coproducts. Let $R=(\text { End } P)^{o p}$. Then $\mathrm{A} \cong R$-Mod. 
Remark 5.2. This, however, is not exactly a reconstruction theorem: the projective generator $P$ is never unique, so we obtain various rings $S=\operatorname{End} P$ such that $\mathrm{A} \cong S$-Mod. Nevertheless, all such rings are regarded as giving the same "noncommutative scheme". So, from the point of view of noncommutative geometry they are the same. By the Gabriel-Rosenberg Reconstruction Theorem, if such $R$ and $S$ are both commutative, then they are necessarily isomorphic.

Two rings $R$ and $S$ are called Morita equivalent if $R$-Mod $\cong S$-Mod.

Example 5.3. For any $\operatorname{ring} R$, the ring $S=M_{n}(R)$ of $n \times n$ matrices over $R$ is Morita equivalent to $R$. (Proof. In the above theorem take $P=R^{\oplus n}$, or use the next theorem.)

The following characterization of Morita equivalence is important. The proof is not hard.

Theorem 5.4. Let $R$ and $S$ be rings. Then the following are equivalent:

i. The categories $R$-Mod and $S$-Mod are equivalent.

ii. There is an $S-R$ bimodule $M$ such that the functor $M \otimes_{R}-: R$-Mod $\rightarrow S$ Mod is an equivalence of categories.

iii. There is a finitely generated projective generator $P$ for $R$-Mod such that $S \cong \operatorname{End} P$.

\section{Appendix: inJeCtive AND PROJECtive obJECts in ABELIAN CATEGories}

We will need to deal with injective and projective objects in the next lecture, so we briefly recall their definition.

An object $P$ in an abelian category is called projective if it has the following lifting property:

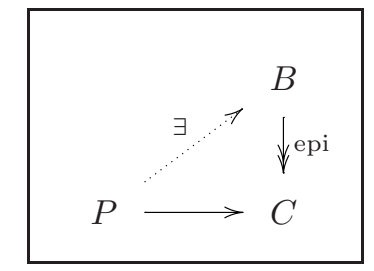

Equivalently, $P$ is projective if the functor $\operatorname{Hom}_{\mathrm{A}}(P,-): \mathrm{A} \rightarrow \mathrm{Ab}$ takes exact sequences in $A$ to exact sequences of abelian groups.

An object $I$ in an abelian category is called injective if it has the following extension property:

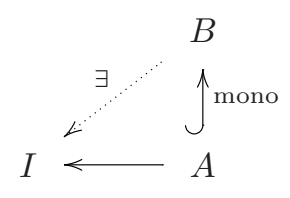


Equivalently, $I$ is injective if the functor $\operatorname{Hom}_{\mathrm{A}}(-, I): \mathrm{A}^{o p} \rightarrow$ Ab takes exact sequences in $\mathrm{A}$ to exact sequences of abelian groups.

We say that A has enough projectives (respectively, enough injectives), if for every object $A$ there exists an epimorphism $P \rightarrow A$ where $P$ is projective (respectively, a monomorphism $A \rightarrow I$ where $I$ is injective).

In a category with enough projectives (respectively, enough injectives) one can always find projective resolutions (respectively, injective resolutions) for objects.

The category $R$-Mod has enough injectives and enough projectives. The categories $\operatorname{PreSh}(C, A b), \operatorname{PreSh}\left(C, R\right.$-Mod), $\mathbf{S h}(C, A b), \operatorname{Sh}(C, R$-Mod $)$, and Quasi-Coh ${ }_{X}$ have enough injectives, but in general they do not have enough projectives.

Exercise. Show that the abelian category of finite abelian groups has no injective or projective object other than 0 . Show that in the category of vector spaces every object is both injective and projective. 


\section{Lecture 2: Chain complexes}

Overview. Various cohomology theories in mathematics are constructed from chain complexes. However, taking the cohomology of a chain complex kills a lot of information contained in that chain complex. So, it is desirable to elevate the cohomological constructions to the chain complex level. In this lecture, we introduce the necessary machinery for doing so. The main tool here is the mapping cone construction, which should be thought of as a homotopy cokernel construction. We discuss in some detail the basic properties of the cone construction; we will see, in particular, how it allows us to construct long exact sequences on the chain complex level, generalizing the usual cohomology long exact sequences.

In practice, one is only interested in chain complexes up to quasi-isomorphism. This leads to the notion of the derived category of chain complexes. The cone construction is well adapted to the derived category. Derived categories provide the correct setting for manipulating chain complexes; for instance, they allow us to construct derived functors on the chain complex level.

\section{Why CHAIN COMPLEXES?}

Chain complexes arise naturally in many areas of mathematics. There are two main sources for (co)chain complexes: chains on spaces and resolutions. We give an example for each.

Chains on spaces. Let $X$ be a topological space. There are various ways to associate a chain complex to $X$. For example, singular chains, cellular chains (if $X$ is a CW complex), simplicial chains (if $X$ is triangulated), de Rham complex (if $X$ is differentiable), and so on. These complexes encode a great deal of topological information about $X$ in terms of algebra (e.g., homology and cohomology).

Observe the following two facts:

- Such constructions usually give rise to a chain complex of free modules.

- Various chain complexes associated to a given space are chain homotopy equivalent (hence, give rise to the same homology/cohomology).

Resolutions. Let us explain this with an example. Let $R$ be a ring, and $M$ and $N$ $R$-modules. Recall how we compute $\operatorname{Tor}_{i}(M, N)$. First, we choose a free resolution

$$
\underbrace{\cdots \rightarrow P^{-2} \rightarrow P^{-1} \rightarrow P^{0}}_{P^{\bullet}} \rightarrow M .
$$

Then, we define

$$
\operatorname{Tor}_{i}(M, N)=H^{-i}\left(P^{\bullet} \otimes N\right) .
$$

Observe the following two facts: 
- The complex $P^{\bullet}$ is a complex of free modules.

- Any two resolutions $P^{\bullet}$ and $Q^{\bullet}$ of $M$ are chain homotopy equivalent (hence Tor is well-defined).

Conclusion. In both examples above, we replaced our object with a chain complex of free modules that was unique up to chain homotopy. We could then extract information about our object by doing algebraic manipulations (e.g., taking homology) on this complex. Therefore, the real object of interest is the (chain homotopy class of) a chain complex (of, say, free modules). Of course, instead of working with the complex itself, one could choose to work with its (co)homology, but one loses some information this way.

Remark 1.1. Complexes of projective modules (e.g, projective resolutions) work equally well as complexes of free modules. Sometimes, we are in a dual situation where complexes of injective modules are more appropriate (e.g., in computing Ext groups).

\section{Chain complexes}

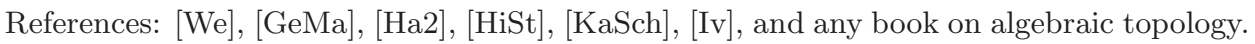

We quickly recall a few definitions. We prefer to work with cohomological indexing, so we work with cochain complexes.

A cochain complex $C$ in an abelian category $\mathrm{A}$ is a sequence of objects in $\mathrm{A}$

$$
\cdots \longrightarrow C^{n-1} \stackrel{d}{\longrightarrow} C^{n} \stackrel{d}{\longrightarrow} C^{n-1} \longrightarrow \cdots, \quad d^{2}=0 .
$$

Such a sequence is in general indexed by $\mathbb{Z}$, but in many applications one works with complexes that are bounded below, bounded above, or bounded on both sides.

A chain map $f: B \rightarrow C$ between two cochain complexes is a sequence of $f^{n}: B^{n} \rightarrow$ $C^{n}$ of morphisms such that the following diagram commutes

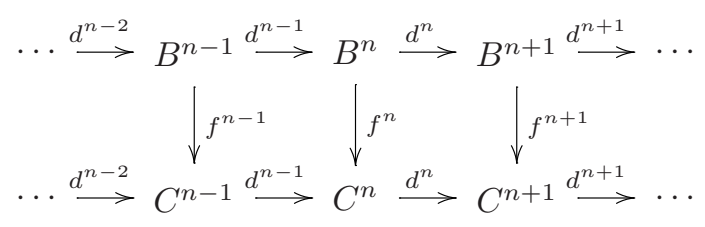

A null homotopy for a chain map $f: B \rightarrow C$ is a sequence $s^{n}: B^{n} \rightarrow C^{n-1}$ such that

$$
f^{n}=s^{n+1} \circ d^{n}+d^{n-1} \circ s^{n}, \quad \forall n
$$


We say two chain maps $f, g: B \rightarrow C$ are chain homotopic if $f-g$ is null homotopic. We say $f: B \rightarrow C$ is a chain homotopy equivalence if there exists $g: C \rightarrow B$ such that $f \circ g$ and $g \circ f$ are chain homotopy equivalent to the corresponding identity maps.

To any chain complex $C$ one can associate the following objects in A:

$$
\begin{aligned}
& Z^{n}(C)=\operatorname{ker}\left(C^{n} \stackrel{d}{\longrightarrow} C^{n+1}\right) \\
& B^{n}(C)=\operatorname{im}\left(C^{n-1} \stackrel{d}{\longrightarrow} C^{n}\right) \\
& H^{n}(C)=Z^{n}(C) / B^{n}(C)
\end{aligned}
$$

Exercise. Show that a chain map $f: B \rightarrow C$ induces morphisms on each of the above objects. In particular, we have induced morphisms $H^{n}(f): H^{n}(B) \rightarrow H^{n}(C)$ for all $n$. Show that if $f$ and $g$ are chain homotopic, then they induce the same map on cohomology. In particular, a chain homotopy equivalence induces isomorphisms on cohomologies.

A chain map $f: B \rightarrow C$ is called a quasi-isomorphism if it induces isomorphisms on all cohomologies. (So every chain homotopy equivalence is a quasi-isomorphism.)

Exercise. When is a chain complex $C$ quasi-isomorphic to the zero complex? (Hint: exact.) When is $C$ chain homotopy equivalent to the zero complex? (Hint: split exact.)

A short exact sequence of chain complexes is a sequence

$$
0 \rightarrow A \rightarrow B \rightarrow C \rightarrow 0
$$

of chain maps which is exact at every $n \in \mathbb{Z}$. Such an exact sequence gives rise to a long exact sequence in cohomology

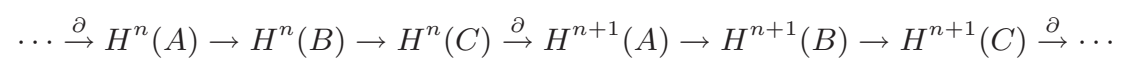

A short exact sequence as above is called pointwise split (or semi-split) if every epimorphism $B^{n} \rightarrow C^{n}$ admits a section, or equivalently, every monomorphism $A^{n} \rightarrow B^{n}$ is a direct summand.

Exercise. Does pointwise split imply that the maps $\partial$ in the above long exact sequence are zero? Is the converse true? (Answer: both implications are false.)

\section{Constructions on Chain COMPleXes}

References: We, GeMa, Ha2, HiSt, KaSch, Iv.

In the following table we list a few constructions that are of great importance in algebraic topology. The left column presents the topological construction, and the right column is the cochain counterpart.

The right column is obtained from the left column as follows: imagine that the spaces are triangulated and translate the topological constructions in terms of the simplices; this gives the chain complex picture. The cochain complex picture is obtained by the appropriate change in indexing (from homological to cohomological). 
The reader is strongly encouraged to do this as an exercise. It is extremely important to pay attention to the orientation of the simplices, and keep track of the signs accordingly.

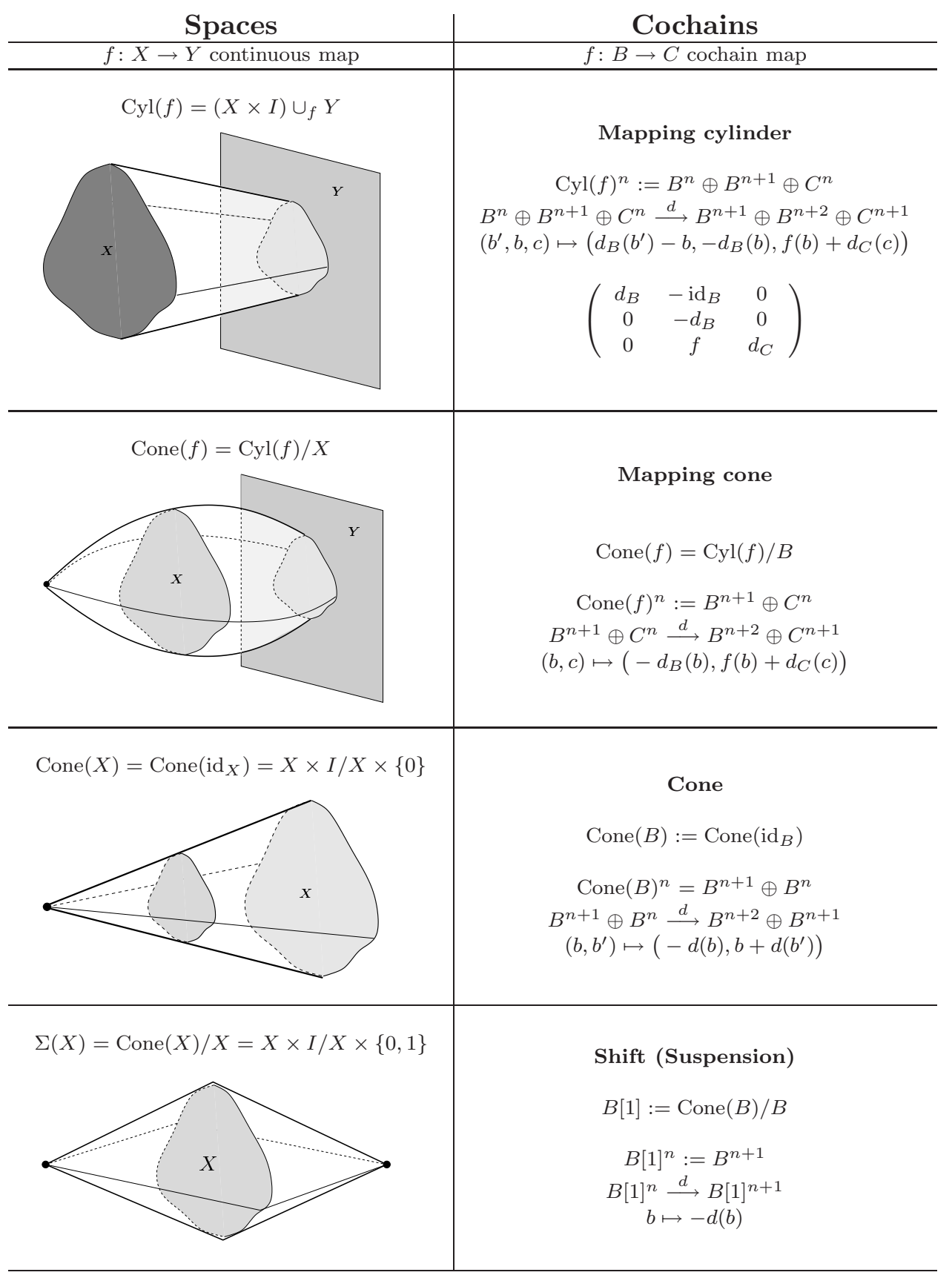


Some history. These constructions were originally used by topologists (e.g., Puppe $\mathrm{Pu}$ ) as a unified way of treating various cohomology theories for topological spaces (the socalled generalized cohomology theories $\mathrm{Ad}]$ ), and soon they became a standard tool in stable homotopy theory. Around the same time, Grothendieck and Verdier developed the same machinery for cochain complexes, to be used in Grothendieck's formulation of duality theory and the Riemann-Roch theorem for schemes Ha2. This led to the invention of derived categories and triangulated categories Ve. As advocated by Grothendieck, it has become more and more apparent over time that working in derived categories is a better alternative to working with cohomology. Nowadays people consider even richer structures

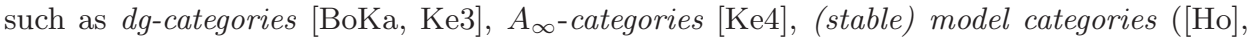
Section 7), etc. The idea is that, the higher one goes in this hierarchy, the more higherorder cohomological information (e.g., Massey products, Steenrod operations, or other cohomology operations) is retained. In these lectures we will not go beyond derived categories.

An important fact. Taking the mapping cone of $f: X \rightarrow Y$ is somewhat like taking cokernels: we are essentially killing the image of $f$, but softly (i.e., we make it contractible). That is why Cone $(f)$ is sometimes called the homotopy cofiber or homotopy cokernel of $f$; see May1, §8.4, especially, the first lemma on page 58.

Proposition 3.1. In the following diagram of cochain complexes, if $g$ and $h$ are chain equivalences, then so is the induced map on the mapping cones

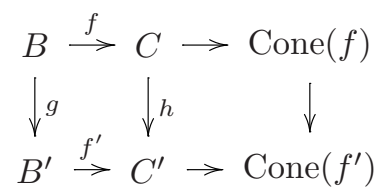

A similar statement is true for topological spaces.

Remark 3.2. The above proposition is still true if we use quasi-isomorphisms instead of chain homotopy equivalences. Similarly, the topological version remains valid if we use weak equivalences (i.e., maps that induce isomorphisms on all homotopy groups) instead of homotopy equivalences.

Exercise. Give an example (in both the topological and chain complex settings) to show that the above proposition is not true if we use the strict cofiber $Y / f(X)$ instead of Cone $(f)$. (Therefore, since we are interested in chain homotopy equivalence classes, or quasi-isomorphism classes, of cochain complexes, Cone $(f)$ is a better-behaved notion than $Y / f(X)$.)

Exercise. Formulate a universal property for the mapping cone construction.

\section{BASIC PROPERTIES OF COFIBER SEQUENCES}

References: We, GeMa], KaSch], Ha2], Ve].

The sequence

$$
B \stackrel{f}{\longrightarrow} C \longrightarrow \text { Cone }(f)
$$


(or any sequence quasi-isomorphic to such a sequence) is called a cofiber sequence. The same definition can be made with topological spaces. We list the basic properties of cofiber sequences.

1. Exact sequence, basic form. An important property of cofiber sequences is that they give rise to long exact sequences. Here is a baby version which is very easy to prove and is left as an exercise.

Proposition 4.1. Let $B \rightarrow C \rightarrow$ Cone $(f)$ be a cofiber sequence. Then the sequence

$$
H^{n}(B) \rightarrow H^{n}(C) \rightarrow H^{n}(\operatorname{Cone}(f))
$$

is exact for every $n$.

Remark 4.2. This is of course part of a long exact sequence

$$
\cdots \stackrel{\partial}{\rightarrow} H^{n}(B) \rightarrow H^{n}(C) \rightarrow H^{n}(\operatorname{Cone}(f)) \stackrel{\partial}{\rightarrow} H^{n+1}(B) \rightarrow H^{n+1}(C) \rightarrow H^{n+1}(\operatorname{Cone}(f)) \stackrel{\partial}{\rightarrow} \cdots
$$

that we will discuss shortly. If we use the fact that a short exact sequence of cochain complexes gives rise to a long exact sequence of cohomology groups, this is easily proven by showing that the cofiber sequence $B \rightarrow C \rightarrow \operatorname{Cone}(f)$ is chain homotopy equivalent to the short exact sequence $B \rightarrow \operatorname{Cyl}(f) \rightarrow \operatorname{Cone}(f)$. The equivalence $C \sim \operatorname{Cyl}(f)$ is given by

$$
\begin{aligned}
& \begin{array}{ccc}
C & \longrightarrow & \operatorname{Cyl}(f) \\
c & \mapsto & (0,0, c)
\end{array} \quad \text { and } \quad \begin{array}{c}
\mathrm{Cyl}(f) \\
B^{n} \oplus B^{n+1} \oplus C^{n}
\end{array} \quad \longrightarrow \quad \begin{array}{c}
C \\
C^{n}
\end{array} \\
& \left(b^{\prime}, b, c\right) \quad \mapsto \quad f\left(b^{\prime}\right)+c .
\end{aligned}
$$

Remark 4.3. We have a similar statement for a cofiber sequence $X \rightarrow Y \rightarrow$ Cone $(f)$ of topological spaces. The corresponding long exact sequence in (co)homology should then be interpreted as the long exact sequence of relative (co)homology groups. More specifically, if $f$ is an inclusion, we have

$$
H_{*}(\operatorname{Cone}(f)) \cong H_{*}(Y, X) \text { and } H^{*}(\operatorname{Cone}(f)) \cong H^{*}(Y, X),
$$

where the right hand sides are relative (co)homology groups.

2. A short exact sequence is a cofiber sequence. We pointed out in Remark 4.2 that a cofiber sequence is chain equivalent (hence quasi-isomorphic) to a pointwise split short exact sequence. The converse is also true.

Proposition 4.4. Consider a short exact sequence of cochain complexes

$$
0 \rightarrow B \stackrel{f}{\rightarrow} C \rightarrow C / B \rightarrow 0 .
$$

Then the natural chain map $\varphi$ : Cone $(f) \rightarrow C / B$ defined by

$$
\begin{array}{ccc}
B^{n+1} \oplus C^{n} & \rightarrow & C^{n} / B^{n} \\
(b, c) & \mapsto & \bar{c}
\end{array}
$$

is a quasi-isomorphism. If the sequence is pointwise split, with $s: C^{n} / B^{n} \rightarrow C^{n}$ a choice of splitting, then $\varphi$ is a chain equivalence. The inverse is given by $\psi: C / B \rightarrow$ Cone $(f)$ 


$$
\begin{array}{ccc}
C^{n} / B^{n} & \rightarrow & B^{n+1} \oplus C^{n} \\
x & \mapsto & (s d(x)-d s(x), s(x)) .
\end{array}
$$

Exercise. Let $f: B \hookrightarrow C$ be an inclusion of $R$-modules, viewed as cochain complexes concentrated in degree 0 . Show that $\varphi$ : $\operatorname{Cone}(f) \rightarrow C / B$ defined above is a chain equivalence if and only if $f$ is split.

3. Iterate of a cofiber sequence. Consider a cofiber sequence

$$
B \stackrel{f}{\longrightarrow} C \stackrel{g}{\longrightarrow} \text { Cone }(f) .
$$

Observe that $g: C \rightarrow$ Cone $(f)$ is a pointwise split inclusion. This implies that the mapping cone of $g$ is naturally chain equivalent to $\operatorname{Cone}(f) / C=B[1]$. More precisely, we have the following commutative diagram:

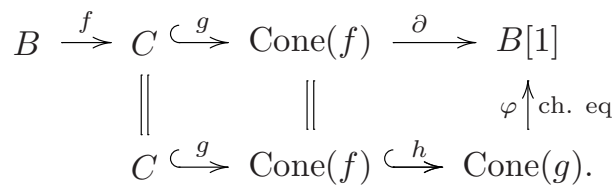

Note that we have natural chain equivalences

$$
\text { Cone }(\partial) \sim \operatorname{Cone}(h) \sim \operatorname{coker}(h)=C[1] .
$$

Indeed, a careful chasing through the above chain equivalences (for which we have given explicit formulas) shows that the sequence

$$
\text { Cone }(f) \stackrel{\partial}{\longrightarrow} B[1] \stackrel{-f[1]}{\longrightarrow} C[1]
$$

is a cofiber sequence. We can now iterate this process forever and produce a long sequence

$$
\ldots \stackrel{\partial[-1]}{\longrightarrow} B \stackrel{f}{\longrightarrow} C \stackrel{g}{\longrightarrow} \operatorname{Cone}(f) \stackrel{\partial}{\longrightarrow} B[1] \stackrel{-f[1]}{\longrightarrow} C[1] \stackrel{-g[1]}{\longrightarrow} \operatorname{Cone}(f)[1] \stackrel{-\partial[1]}{\longrightarrow} \cdots
$$

in which every three consecutive terms form a cofiber sequence.

Remark 4.5. A similar long exact sequence can be constructed for a map $f: X \rightarrow Y$ of topological spaces, and it is called a Puppe sequence (or cofiber sequence [May1] §8.4). A Puppe sequence, however, only extends to the right. The reason for this is that the suspension functor on the category of topological spaces is not invertible (as opposed to the shift functor for cochain complexes). The Puppe sequence can be used, among other things, to give a natural construction of the long exact (co)homology sequence of a pair.

4. Long exact sequence of a cofiber sequence. Applying Proposition 4.1 to the above long exact sequence of chain complexes, and observing that $H^{i}(B[n])=$ $H^{i+n}(B)$, we obtain a long exact sequence of cohomology groups

$$
\cdots \stackrel{\partial}{\rightarrow} H^{n}(B) \rightarrow H^{n}(C) \rightarrow H^{n}(\operatorname{Cone}(f)) \stackrel{\partial}{\rightarrow} H^{n+1}(B) \rightarrow H^{n+1}(C) \rightarrow H^{n+1}(\operatorname{Cone}(f)) \stackrel{\partial}{\rightarrow} \cdots
$$


Moral. By exploiting the notion of cone of a map in a systematic way, we can elevate many basic cohomological constructions to the level of chain complexes. For this to work conveniently, one needs to invert chain equivalences so that one can treat such morphisms as isomorphisms. Indeed, for various reasons, inverting only chain equivalences is usually not enough. For example, recall that one of our motivations for working with chain complexes was that we wanted to be able to replace an object $M \in \mathrm{A}$ with a better behaved resolution (projective or injective) of it. Since the resolution map is only a quasi-isomorphism and not a chain equivalence in general, it is only after inverting quasi-isomorphisms that we can regard an $M \in \mathrm{A}$ and its resolution as the "same".

\section{Derived Categories}

References: We, GeMa], [Ha2], KaSch, [Iv, $\mathrm{Ve}$, Ke1, [Ke2].

Let $\mathrm{A}$ be an abelian category.

The homotopy category $\mathcal{K}(\mathrm{A})$ is the category obtained by inverting all chain equivalences in the category $\mathrm{Ch}(\mathrm{A})$ of chain complexes. More precisely, there is a natural functor $\mathrm{Ch}(\mathrm{A}) \rightarrow \mathcal{K}(\mathrm{A})$ which has the following universal property:

If a functor $F: \mathrm{Ch}(\mathrm{A}) \rightarrow \mathrm{C}$ sends chain equivalences to isomorphisms, then

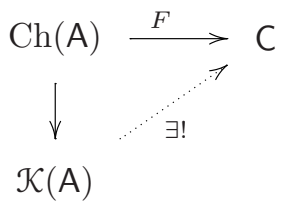

$\mathcal{K}(\mathrm{A})$

If in the above definition we replace 'chain equivalence' by 'quasi-isomorphism', we arrive at the definition of the derived category $\mathcal{D}(\mathrm{A})$. We list the basic properties of $\mathcal{K}(\mathrm{A})$ and $\mathcal{D}(\mathrm{A})$.

1. Explicit construction of $\mathcal{K}(\mathrm{A})$. We can, alternatively, define the homotopy category $\mathcal{K}(\mathrm{A})$ to be the category whose objects are the ones of $\mathrm{Ch}(\mathrm{A})$ and whose morphisms are defined by

$$
\operatorname{Hom}_{\mathcal{K}(\mathbf{A})}(B, C):=\operatorname{Hom}_{\mathrm{Ch}(\mathrm{A})}(B, C) / N
$$

where $N$ stands for the group of null homotopic maps. (Exercise. Show that this category satisfies the required universal property.) This, in particular, implies that $\mathcal{K}(\mathrm{A})$ is an additive category. 
2. Cohomology functors. The cohomology functors $H^{n}: \mathrm{Ch}(\mathrm{A}) \rightarrow \mathrm{A}$ factor through $\mathcal{K}(\mathrm{A})$ and $\mathcal{D}(\mathrm{A})$ :

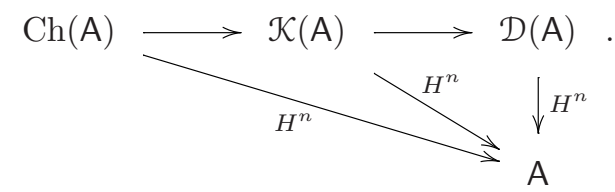

3. Explicit construction of $\mathcal{D}(\mathrm{A})$. The objects of $\mathcal{D}(\mathrm{A})$ can again be taken to be the ones of $\mathrm{Ch}(\mathrm{A})$. Description of morphisms in $\mathcal{D}(\mathrm{A})$ is, however, slightly more involved than the case of $\mathcal{K}(\mathrm{A})$. It requires the notion of calculus of fractions in a category, which we will not get into for the sake of brevity.

Just to give an idea how this works, first we observe that $\mathcal{D}(A)$ can, equivalently, be defined by inverting quasi-isomorphisms in $\mathcal{K}(\mathrm{A})$. The class of quasiisomorphisms in $\mathcal{K}(\mathrm{A})$ satisfies the axioms of the calculus of fractions (GeMa, Definition III.2.6), and this allows one to give an explicit description of morphisms in $\mathcal{D}(\mathrm{A})$ (see GeMa, Section III.2, Ha2, Section I.3, or We, Section 10.3). A consequence of this is that, for every morphism $f: B \rightarrow C$ in $\mathcal{D}(\mathrm{A})$, one can find morphisms $s, t, g$ and $h$ in $\mathcal{K}(\mathrm{A})$ such that $s$ and $t$ are quasi-isomorphisms and the following diagrams commute in $\mathcal{D}(\mathrm{A})$ :
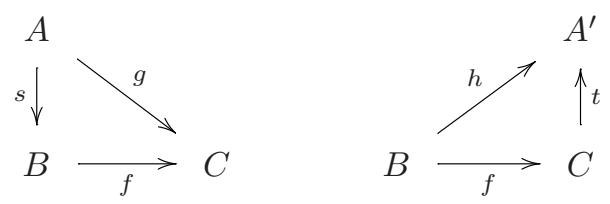

Also, for any two morphisms $f, f^{\prime}: B \rightarrow C$, now in $\mathcal{K}(\mathrm{A})$, which become equal in $\mathcal{D}(\mathrm{A})$, there are $s$ and $t$ as above such that $f \circ s=f^{\prime} \circ s$ and $t \circ f=t \circ f^{\prime}$.

4. Cofiber sequences. There is a notion of cofiber sequence in both $\mathcal{K}(\mathrm{A})$ and $\mathcal{D}(\mathrm{A})$. Every morphism $f: B \rightarrow C$ fits in a sequence $\star$ as in page 19 in which every three consecutive terms form a cofiber sequence.

\section{VARIATIONS ON THE THEME OF DERIVED CATEGORIES}

References: We, GeMa, $\mathrm{Ha2}$, KaSch, Iv, Ke1, Ke2].

To an abelian category A we can associate various types of homotopy or derived categories by imposing certain boundedness conditions on the chain complexes in question.

The bounded below derived category $\mathcal{D}^{+}(\mathrm{A})$ is the category obtained from inverting the quasi-isomorphisms in the category of bounded below chain complexes 
$\mathrm{Ch}^{+}(\mathrm{A})$. Equivalently, $\mathcal{D}^{+}(\mathrm{A})$ is obtained from inverting the quasi-isomorphisms in the homotopy category $\mathcal{K}^{+}(\mathrm{A})$ of $\mathrm{Ch}^{+}(\mathrm{A})$.

In the same way one can define bounded derived categories $\mathcal{D}^{-}(\mathrm{A})$ and $\mathcal{D}^{b}(\mathrm{~A})$, where $b$ stands for bounded on two sides.

Remark 6.1. One can use the calculus of fractions in $\mathcal{K}^{+}(\mathrm{A})$ (respectively, $\mathcal{K}^{-}(\mathrm{A})$, $\mathcal{K}^{b}(\mathrm{~A})$ ) to give a description of morphisms in $\mathcal{D}^{+}(\mathrm{A})$ (respectively, $\mathcal{D}^{-}(\mathrm{A}), \mathcal{D}^{b}(\mathrm{~A})$ ). It follows that each of these bounded derived categories can be identified with a full subcategory of $\mathcal{D}(\mathrm{A})$. In particular, $\mathcal{D}^{b}(\mathrm{~A})=\mathcal{D}^{+}(\mathrm{A}) \cap \mathcal{D}^{-}(\mathrm{A})$. We will say more on this in the next lecture.

There is an alternative way of computing morphisms in bounded derived categories using injective or projective resolutions. It is summarized in the following theorems.

Theorem 6.2 (GeMa, $\S$ III.5.21, We, Theorem 10.4.8). Let $\mathcal{J}^{+}(\mathrm{A}) \subset \mathcal{K}^{+}(\mathrm{A})$ be the full subcategory consisting of complexes whose terms are all injective. Then the composition

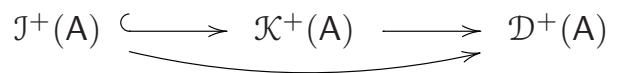

is fully faithful. It is an equivalence if A has enough injectives. The same thing is true if we replace + by - and injective by projective:

$$
\mathcal{P}^{-}(\mathrm{A}) \longleftrightarrow \mathcal{K}^{-}(\mathrm{A}) \longrightarrow \mathcal{D}^{-}(\mathrm{A})
$$

The (first part of the) above theorem says that, if $I$ and $J$ are bounded below complexes of injective objects, then we have

$$
\operatorname{Hom}_{\mathcal{D}(\mathrm{A})}(J, I)=\operatorname{Hom}_{\mathcal{K}(\mathrm{A})}(J, I) .
$$

(This is actually true if $J$ is an arbitrary bounded below complex; see [We, Corollary 10.4.7). In particular, if $I$ and $J$ are quasi-isomorphic, then they are chain equivalent. This is good news, because we have seen that computing Hom is much easier in $\mathcal{K}(\mathrm{A})$. Now if $\mathrm{A}$ has enough injectives, it can be shown that for every bounded $C$, there exists a bounded below complex of injectives $I$, and a quasi-isomorphism $C \stackrel{\sim}{\longrightarrow}$. This is called an injective resolution for $C$. So, by virtue of the above fact, injective resolutions can be used to compute morphisms in derived categories:

$$
\operatorname{Hom}_{\mathcal{D}(\mathrm{A})}(B, C)=\operatorname{Hom}_{\mathcal{K}(\mathrm{A})}(B, I) .
$$

Exercise. Set $\operatorname{Hom}_{\mathcal{D}(\mathrm{A})}^{i}(B, C):=\operatorname{Hom}(B, C[i])$. Let $B$ and $C$ be objects in A, viewed as complexes concentrated in degree 0 . Assume $A$ has enough injectives. Show that

$$
\operatorname{Hom}_{\mathcal{D}(\mathrm{A})}^{i}(B, C) \cong \operatorname{Ext}^{i}(B, C)
$$


Use the second part of the theorem to give a way of computing Ext groups using a projective resolution for $C$, if they exist. Compute $\operatorname{Hom}_{\mathcal{K}(\mathrm{A})}^{i}(B, C)$ and compare it with $\operatorname{Hom}_{\mathcal{D}(\mathrm{A})}^{i}(B, C)$.

Exercise. Give an example of a non-zero morphism in $\mathcal{D}(\mathrm{A})$, with $\mathrm{A}$ your favorite abelian category, which induces zero maps on all cohomologies.

\section{DeRIVED FunCtors}

References: We, GeMa, $\underline{\mathrm{Ha} 2}$, KaSch, 피, Ke1, Ke2.

To keep the lecture short, we will skip the very important topic of derived functors and confine ourselves to an example: the derived tensor $\stackrel{L}{\otimes}$. The reader is encouraged to consult the given references for the general discussion of derived functors, especially the all important derived hom RHom.

Let $\mathrm{A}=R$-Mod. The idea is that we want to have a notion of tensor product for chain complexes which is well defined on $\mathcal{D}(A)$.

The usual tensor product of chain complexes (Definition [We, §2.7.1) does not pass to derived categories. This is because tensor product is not exact. More precisely, if $A \rightarrow A^{\prime}$ is a quasi-isomorphism, then $A \otimes B \rightarrow A^{\prime} \otimes B$ may no longer be. (For a counterexample, let $A$ be a short exact sequence, $A^{\prime}=0$, and $B$ a complex concentrated in degree 0 .)

The usual tensor product of chain complexes DOES pass to homotopy categories. This is because a null homotopy $s$ for a chain map $A \rightarrow A^{\prime}$ gives rise to a null homotopy $s \otimes B$ for $A \otimes B \rightarrow A^{\prime} \otimes B$ (exercise).

In particular, taking all complexes to be (bounded-above) complexes of projective modules, we get a well-defined tensor product $-\otimes-: \mathcal{P}^{-}(A) \times \mathcal{P}^{-}(A) \rightarrow \mathcal{P}^{-}(A)$. Since $R$-Mod has enough projectives, we obtain, via the equivalence $\mathcal{P}^{-}(\mathrm{A}) \cong$ $\mathcal{D}^{-}$(A) of Theorem 6.2. the desired tensor product on the bounded above derived category:

$$
-\stackrel{L}{\otimes}-: \mathcal{D}^{-}(\mathrm{A}) \times \mathcal{D}^{-}(\mathrm{A}) \rightarrow \mathcal{D}^{-}(\mathrm{A})
$$

More explicitly, $A \stackrel{L}{\otimes} B$ is defined to be $P \otimes Q$, where $P \rightarrow A$ and $Q \rightarrow B$ are certain chosen projective resolutions. (In fact, it is enough to resolve only one of $A$ or $B$.)

Exercise. Show that if $M, N \in \mathrm{A}$ are viewed as complexes concentrated in degree 0 , then

$$
H^{-i}(M \stackrel{L}{\otimes} N) \cong \operatorname{Tor}_{i}(M, N)
$$




\section{Lecture 3: Triangulated categories}

Overview. The main properties of the cone construction for chain complexes can be formalized into a set of axioms. This leads to the notion of a triangulated category, the main topic of this lecture. A triangulated category is an additive category in which there is an abstract notion of mapping cone. The cohomology functors on chain complexes can also be studied at an abstract level in a triangulated category.

The main example of a triangulated category is the derived category $\mathcal{D}(A)$ of chain complexes in an abelian category $A$. Using a t-structure on a triangulated category, we can produce an abelian category, called the heart of the $t$-structure. For example, there is a standard $t$-structure on $\mathcal{D}(\mathrm{A})$ whose heart is $\mathrm{A}$. The $t$ structure is not unique, and by varying it we can produce new abelian categories. We show how using a torsion theory on an abelian category $A$ we can produce a new $t$-structure on $\mathcal{D}(\mathrm{A})$. The heart of this $t$-structure is then a new abelian category $\mathrm{B}$. For example, this method has been used in noncommutative geometry to "deform" the abelian category $\mathrm{A}$ of coherent sheaves on a torus $X$. The new abelian category $B$ can then be thought of as the category of coherent sheaves on a "noncommutative deformation" of $X$, a noncommutative torus.

\section{Triangulated Categories}

References: $\mathrm{We}, \mathrm{GeMa}, \mathrm{Ha2}, \mathrm{Ne}, \mathrm{BeBeDe}, \mathrm{KaSch}, \mathrm{Iv}, \mathrm{Ve}, \mathrm{Ke} 1, \mathrm{Ke} 2$.

We formalize the main properties of the mapping cone construction and define triangulated categories.

Let $\mathcal{T}$ be an additive category equipped with an auto-equivalence $X \mapsto X[1]$ called shift (or translation).

By a triangle in $\mathcal{T}$ we mean a sequence $X \rightarrow Y \rightarrow Z \rightarrow X[1]$. We sometimes write this as

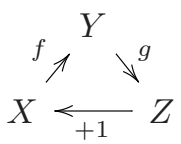

A triangulation on $\mathcal{T}$ is a collection of triangles, called exact (or distinguished) triangles, satisfying the following axioms:

TR1. a) $X \stackrel{\text { id }}{\longrightarrow} X \longrightarrow 0 \longrightarrow X[1]$ is an exact triangle.

b) Any morphism $f: X \rightarrow Y$ is part of an exact triangle.

c) Any triangle isomorphic to an exact triangle is exact.

TR2. $X \stackrel{f}{\rightarrow} Y \stackrel{g}{\rightarrow} Z \stackrel{h}{\rightarrow} X[1]$ exact $\Leftrightarrow Y \stackrel{g}{\rightarrow} Z \stackrel{h}{\rightarrow} X[1] \stackrel{-f[1]}{\rightarrow} Y[1]$ exact.

TR3. In the diagram 


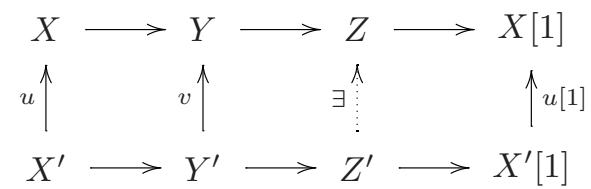

if the horizontal rows are exact, and the left square commutes, then the dotted arrow can be filled (not necessarily uniquely) to make the diagram commute.

TR4 (short imprecise version). Given $f: X \rightarrow Y$ and $g: Y \rightarrow Z$, we have the following commutative diagram in which every pair of same color arrows is part of an exact triangle

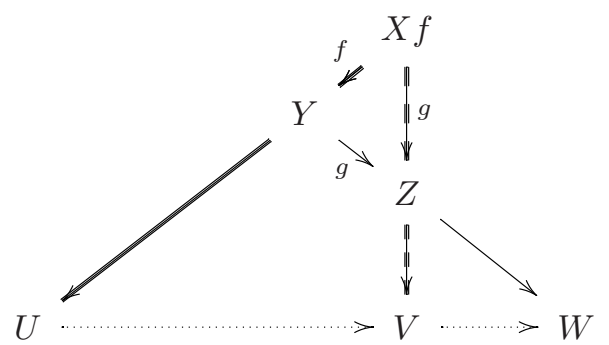

Idea: " $(Z / X) /(Y / X)=Z / Y)$ ". See Appendix II for a more precise statement.

A triangulated category is an additive category $\mathcal{T}$ equipped with an autoequivalence $X \mapsto X[1]$ and a triangulation.

A triangle functor (or a exact functor) ${ }^{1}$ is a functor between triangulated categories which commutes with the translation functors (up to natural transformation) and sends exact triangles to exact triangles.

Remark 1.1. A triangulated category can be thought of as a category in which there are well-behaved notions of homotopy kernel and homotopy cokerne (hence also various other types of homotopy limits and colimits).

Some immediate consequences. Let $\mathcal{T}$ be a triangulated category, and let $X \rightarrow Y \rightarrow Z \rightarrow X[1]$ be an exact triangle in $\mathcal{T}$. Then, the following are true:

0. The opposite category $\mathcal{T}^{o p}$ is naturally triangulated.

1. Any length three portion of the sequence

$$
\cdots \longrightarrow Z[-1] \stackrel{-h[-1]}{\longrightarrow} X \stackrel{f}{\longrightarrow} Y \stackrel{g}{\longrightarrow} Z \stackrel{h}{\longrightarrow} X[1] \stackrel{-f[1]}{\longrightarrow} Y[1] \stackrel{-g[1]}{\longrightarrow} Z[1] \stackrel{-h[1]}{\longrightarrow} \cdots
$$

\footnotetext{
${ }^{1}$ This is not a very good terminology, because there is also a notion of $t$-exact functor with respect to a $t$-structure.

${ }^{2}$ Indeed not quite well-behaved (read, functorial), essentially due to the fact that the morphism whose existence is required in TR3 may not be unique. This turns out to be problematic. A way to remedy this is to work with DG categories; see BoKa.
} 
is an exact triangle.

2. In the above exact sequence, any two consecutive morphisms compose to zero. Proof. Enough to check $g \circ f=0$. Apply TR1. $a$ and TR3 to

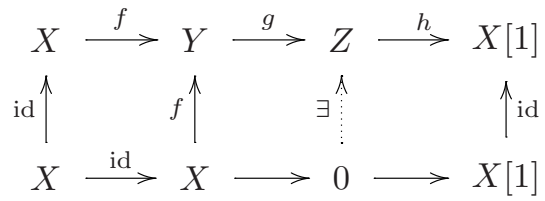

3. Let $T$ be any object. Then,

$$
\operatorname{Hom}(T, X) \rightarrow \operatorname{Hom}(T, Y) \rightarrow \operatorname{Hom}(T, Z)
$$

is exact. Therefore, the long sequence of (1) gives rise to a long exact sequence of abelian groups. The same thing is true for

$$
\operatorname{Hom}(X, T) \rightarrow \operatorname{Hom}(Y, T) \rightarrow \operatorname{Hom}(Z, T) .
$$

Remark 1.2. In fact, TR3 and half of TR2 follow from the rest of the axioms. For this, see May2]. Also see [Ne for another formulation of TR4; especially, Remark 1.3.15 and Proposition 1.4.6.

The main example. The proof of the following theorem can be found in GeMa or KaSch. (We have sketched some of the ideas in Lecture 2.)

Theorem 1.3. Let $\mathrm{A}$ be an abelian category. Then $\mathcal{K}(\mathrm{A})$ and $\mathcal{D}(\mathrm{A})$ are triangulated categories.

In both cases, the distinguished triangles are the ones obtained from cofiber sequences. Equivalently, the distinguished triangles are the ones obtained from the pointwise split short exact sequences. (To see the equivalence, note that one implication follows from Proposition 4.4 of Lecture 2. The other follows from the fact that the cofiber sequence $B \rightarrow C \rightarrow \operatorname{Cone}(f)$ is chain homotopy equivalent to the pointwise split short exact sequence $B \rightarrow \mathrm{Cyl}(f) \rightarrow \mathrm{Cone}(f)$; see Remark 4.2 of Lecture 2.) In the case of $\mathcal{D}(\mathrm{A})$ we get the same triangulation if we take all short exact sequences. This also follows from Proposition 4.4 of Lecture 2.

The following proposition allows us to produce more triangulated categories from the above basic ones.

Proposition 1.4. Let $\mathrm{A}$ be an abelian category, and let $\mathrm{C}$ be a full additive subcategory of $\mathrm{Ch}(\mathrm{A})$. Let $\mathcal{K} \subset \mathcal{K}(\mathrm{A})$ be the corresponding quotient category and $\mathcal{D}$ the localization of $\mathcal{K}$ with respect to quasi-isomorphisms. Assume $\mathcal{C}$ is closed under translation, quasi-isomorphisms, and forming mapping cones. Then $\mathcal{K}$ and $\mathcal{D}$ are triangulated categories and we have fully faithful triangle functors

$$
\mathcal{K} \rightarrow \mathcal{K}(\mathrm{A})
$$




$$
\mathcal{D} \rightarrow \mathcal{D}(\mathrm{A}) .
$$

Proof. The proof in the case of $\mathcal{K}$ is straightforward. The case of $\mathcal{D} \rightarrow \mathcal{D}(\mathrm{A})$ follows easily from the existence of calculus of fractions on $\mathcal{K}(\mathrm{A})$. (Our discussion of calculus of fractions in Lecture 2 is enough for this.)

Exercise. Show that $\mathcal{D}^{-}(\mathrm{A})$ can, equivalently, be defined by inverting the quasiisomorphisms in the category $\mathcal{C}$ of chain complexes with bounded above cohomology. The similar statement is true in the case of $\mathcal{D}^{+}(\mathrm{A})$ and $\mathcal{D}^{b}(\mathrm{~A})$. (Hint: construct a right inverse to the inclusion $\iota: \mathrm{Ch}^{-}(\mathrm{A}) \hookrightarrow \mathcal{C}$ by choosing an appropriate truncation of each complex in C; see page 29 to learn how to truncate. Show that this becomes an actual inverse to $\iota$ when we pass to derived categories.)

Corollary 1.5. We have the following fully faithful triangle functors:

$$
\begin{aligned}
& \mathcal{K}^{-}(\mathrm{A}), \mathcal{K}^{+}(\mathrm{A}), \mathcal{K}^{b}(\mathrm{~A}) \hookrightarrow \mathcal{K}(\mathrm{A}), \\
& \mathcal{D}^{-}(\mathrm{A}), \mathcal{D}^{+}(\mathrm{A}), \mathcal{D}^{b}(\mathrm{~A}) \hookrightarrow \mathcal{D}(\mathrm{A}) .
\end{aligned}
$$

Proof. This is an immediate corollary of the previous proposition. (We also need to use the previous exercise.)

Important examples to keep in mind:

1. $\mathrm{A}=R$-Mod.

2. $\mathrm{A}=$ sheaves of $R$-modules over a topological space.

3. A $=$ presheaves of $R$-modules over a topological space.

4. $\mathrm{A}=$ sheaves of $\mathcal{O}_{X}$-modules on a scheme $X$.

5. $\mathrm{A}=$ quasi-coherent sheaves on a scheme $X$.

\section{Cohomological Functors}

References: We, [GeMa], BeBeDe] [Ha2], KaSch, $\mathrm{Ve}$.

Let $\mathcal{T}$ be a triangulated category and $\mathrm{A}$ an abelian category. An additive functor $H: \mathcal{T} \rightarrow \mathrm{A}$ is called a cohomological functor if for every exact triangle $X \rightarrow$ $Y \rightarrow Z \rightarrow X[1]$

$$
H(X) \rightarrow H(Y) \rightarrow H(Z) \text { is exact in } \mathrm{A} .
$$

If we set $H^{n}(X):=H(X[n])$, we obtain the following long exact sequence:

$\cdots \longrightarrow H^{n-1}(Z) \rightarrow H^{n}(X) \rightarrow H^{n}(Y) \rightarrow H^{n}(Z) \rightarrow H^{n+1}(X) \rightarrow H^{n+1}(Y) \rightarrow H^{n+1}(Z) \rightarrow \cdots$

Example 2.1. 
1. Let $\mathcal{T}$ be any of $\mathcal{K}^{*}(\mathrm{~A})$ or $\mathcal{D}^{*}(\mathrm{~A}), *=\emptyset,-,+, b$. Then the functor $H: \mathcal{T} \rightarrow \mathrm{A}$, $X \mapsto H^{0}(X)$ is a cohomological functor.

2. For any $T, \operatorname{Hom}(T,-): \mathcal{T} \rightarrow \mathrm{A}$ is a cohomological functor. Similarly, $\operatorname{Hom}(-, T): \mathcal{T} \rightarrow \mathrm{A}^{o p}$ is a cohomological functor.

Exercise. Show that (1) is a special case of (2).

3. Abelian CAtegories inside triangulated CATEgories; $t$-STRuCtures

References: GeMa, KaSch, BeBeDe.

The triangulated categories associated to an abelian category A contain A as a full subcategory. More precisely,

Proposition 3.1. Let $\mathcal{T}$ be any of $\mathcal{K}^{*}(\mathrm{~A})$ or $\mathcal{D}^{*}(\mathrm{~A}), *=\emptyset,-,+, b$. Then the functor $\mathrm{A} \rightarrow \mathcal{T}$ defined by

$$
A \quad \mapsto \quad \cdots \rightarrow 0 \rightarrow A \rightarrow 0 \rightarrow \cdots
$$

( $A$ is sitting in degree zero) is fully faithful.

Observe that this is not the only abelian subcategory of $\mathcal{T}$. For example, we have $\mathbb{Z}$ many copies of $A$ in $\mathcal{T}$ (simply take shifts of the above embedding). We may also have abelian categories in $\mathcal{T}$ that are not isomorphic to $A$.

As we saw above, the triangulated structure of $\mathcal{T}$ is not sufficient to recover the abelian subcategory $A$. What extra structure do we need on $\mathcal{T}$ in order to reconstruct $A$ ?

A $t$-structure on a triangulated category $\mathcal{T}$ is a pair $(\mathcal{T} \leq 0, \mathcal{T} \geq 0)$ of saturated (i.e., closed under isomorphism) full subcategories such that:

t1. If $X \in \mathcal{T} \leq 0, Y \in \mathcal{T} \geq 1$, then $\operatorname{Hom}(X, Y)=0$.

t2. $\mathcal{T} \leq 0 \subseteq \mathcal{T} \leq 1$, and $\mathcal{T} \geq 1 \subseteq \mathcal{T} \geq 0$.

t3. For every $X \in \mathcal{T}$, there is an exact triangle

$$
A \rightarrow X \rightarrow B \rightarrow A[1]
$$

such that $A \in \mathcal{T} \leq 0$ and $B \in \mathcal{T} \geq 1$.

Notation: $\mathcal{T}^{\leq n}=\mathcal{T}^{\leq 0}[-n]$ and $\mathcal{T}^{\geq n}=\mathcal{T}^{\geq 0}[-n]$.

Main example. Let $\mathcal{T}=\mathcal{D}(A)$. Set

$$
\begin{aligned}
& \mathcal{T} \leq 0=\left\{X \in \mathcal{D}(\mathrm{A}) \mid H^{i}(X)=0, \forall i>0\right\} \\
& \mathcal{T} \geq 0=\left\{X \in \mathcal{D}(\mathrm{A}) \mid H^{i}(X)=0, \forall i<0\right\}
\end{aligned}
$$

The proof that this is a $t$-structure is not hard. The axioms (t1) and (t2) are straightforward. To verify (t3), we define truncation functors

$$
\tau_{\leq 0}: \mathcal{T} \rightarrow \mathcal{T} \leq 0
$$




$$
\tau_{\geq 1}: \mathcal{T} \rightarrow \mathcal{T}^{\geq 1}
$$

by

$$
\begin{gathered}
\tau_{\leq 0}(X):=\quad \cdots \rightarrow X^{-2} \rightarrow X^{-1} \rightarrow \operatorname{ker} d \rightarrow 0 \rightarrow 0 \rightarrow \cdots \\
\tau_{\geq 1}(X):=X / \tau_{\leq 0}(X)=\quad \cdots \rightarrow 0 \rightarrow 0 \rightarrow X_{0} / \operatorname{ker} d \rightarrow X^{1} \rightarrow X^{2} \rightarrow \cdots
\end{gathered}
$$

It is easy to see that

$$
\tau_{\leq 0} X \rightarrow X \rightarrow \tau_{\geq 1} X \rightarrow \tau_{\leq 0} X[1]
$$

is exact (Lecture 2, Proposition 4.4). So, we can take $A=\tau_{\leq 0} X$ and $B=\tau_{\geq 1} X$.

Remark 3.2. This $t$-structure induces $t$-structures on each of $\mathcal{D}^{-,+, b}(\mathrm{~A})$.

Exercise. Show that this does not give a $t$-structure on $\mathcal{K}(\mathrm{A})$. (Hint: show that (t1) fails by taking $X=Y$ to be an exact complex that is not contractible, e.g., a non-split short exact sequence.)

The truncation functors discussed above can indeed be defined for any $t$-structure.

Proposition 3.3 (BeBeDe, $\S 1.3$ ). Let $\mathcal{T}$ be a triangulated category equipped with at-structure $(\mathcal{T} \leq 0, \mathcal{T} \geq 0)$.

i. The inclusion $\mathcal{T} \leq n \hookrightarrow \mathcal{T}$ admits a right adjoint $\tau_{\leq n}: \mathcal{T} \rightarrow \mathcal{T} \leq n$, and the inclusion $\mathcal{T} \geq n \hookrightarrow \mathcal{T}$ admits a left adjoint $\tau_{\geq n}: \mathcal{T} \rightarrow \mathcal{T} \geq n$.

ii. For every $X \in \mathcal{T}$ and every $n \in \mathbb{Z}$, there is a unique $d$ that makes the triangle

$$
\tau_{\leq n} X \longrightarrow X \longrightarrow \tau_{\geq n+1} X \stackrel{d}{\longrightarrow} \tau_{\leq n} X[1]
$$

exact.

Remark 3.4. An aisle in a triangulated category is a full saturated subcategory $\mathfrak{U}$ that is closed under extensions, $\mathcal{U}[1] \subseteq \mathcal{U}$, and such that $\mathcal{U} \rightarrow \mathcal{T}$ admits a right adjoint. For example, $\mathcal{U}=\mathcal{T} \leq 0$ is an aisle. Conversely, any aisle $\mathcal{U}$ is equal to $\mathcal{T} \leq 0$ for a unique $t$-structure $(\mathcal{T} \leq 0, \mathcal{T} \geq 0)$; see $\underline{\mathrm{KeVO}}$.

Proposition 3.5 ( $\mathrm{BeBeDe}, \S 1.3)$. Let $a \leq b$. Then $\tau_{\geq a}$ and $\tau_{\leq b}$ commute, in the sense that

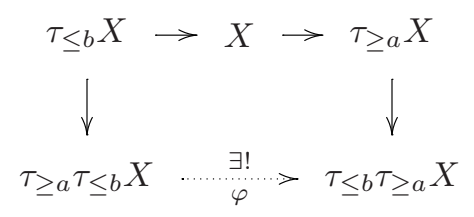

is commutative. Furthermore, $\varphi$ is necessarily an isomorphism.

We set $\tau_{[a, b]}:=\tau_{\geq a} \tau_{\leq b} X$. We call $\mathcal{T} \leq 0 \cap \mathcal{T} \geq 0$ the heart (or core) of the $t$-structure.

Theorem 3.6 (BeBeDe, $\S 1.3)$. The heart $\mathrm{C}:=\mathcal{T} \leq 0 \cap \mathcal{T} \geq 0$ is an abelian category. The functor $H^{0}:=\tau_{[0,0]}: \mathcal{T} \rightarrow C$ is a cohomological functor.

Example 3.7. The heart of the standard $t$-structure on $\mathcal{D}(\mathrm{A})$ is $\mathrm{A}$, and the functor $\tau_{[0,0]}$ is the usual $H^{0}$. 
Remark 3.8. In the above theorem, $\mathcal{T}$ may not be equivalent to any of $\mathcal{D}^{\emptyset,-,+, b}(\mathrm{C})$.

The moral of the story is that, by varying $t$-structures inside a triangulated category (e.g., $\mathcal{D}(\mathrm{A})$ ), we can produce new abelian categories.

\section{Producing new abelian Categories}

Reference: HaReSm.

One can use $t$-structures to produce new abelian categories out of given ones. This technique appeared for the first time in BeBeDe where they alter the $t$ structures on various derived categories of sheaves on a space to produce new abelian categories of perverse sheaves (see loc. cit. $\S 2$ and also Theorem 1.4.10).

Another way to produce $t$-structures is via torsion theories. For an application of this in noncommutative geometry see $[\mathrm{PO}]$.

A torsion theory on an abelian category $A$ is a pair $(\mathbb{T}, \mathbb{F})$ of full additive subcategories of A such that:

- For every $T \in \mathbb{T}$ and $F \in \mathbb{F}$, we have $\operatorname{Hom}(T, F)=0$.

- For every $A \in \mathrm{A}$, there is a (necessarily unique) exact sequence

$$
0 \rightarrow T \rightarrow A \rightarrow F \rightarrow 0, \quad T \in \mathbb{T}, F \in \mathbb{F} .
$$

Example 4.1. Let $\mathbf{A}=\mathbf{A} \mathbf{b}$ be abelian categories. Take $\mathbb{T}$ to be the torsion abelian groups and $\mathbb{F}$ the torsion-free abelian groups.

Exercise. Prove that for a torsion theory $(\mathbb{T}, \mathbb{F})$ we have $\mathbb{T}^{\perp}=\mathbb{F}$ and ${ }^{\perp} \mathbb{F}=\mathbb{T}$, that is,

$$
\begin{aligned}
& \mathbb{F}=\{F \in \mathrm{A} \mid \forall T \in \mathbb{T}, \operatorname{Hom}(T, F)=0\}, \\
& \mathbb{T}=\{T \in \mathrm{A} \mid \forall F \in \mathbb{F}, \operatorname{Hom}(T, F)=0\} .
\end{aligned}
$$

Out of a given torsion theory $(\mathbb{T}, \mathbb{F})$ on an abelian category A we can construct a new abelian category $B$ in which the roles of $\mathbb{T}$ and $\mathbb{F}$ are interchanged.

We construct $B$ as follows. Consider the following $t$-structure on $\mathcal{D}=\mathcal{D}(\mathrm{A})$ :

$$
\begin{aligned}
& \mathbb{D} \leq 0=\left\{B \in \mathcal{D}^{\leq 0} \mid H^{0}(B) \in \mathbb{T}\right\}, \\
& \mathbb{D}^{\geq 0}=\left\{B \in \mathcal{D}^{\geq-1} \mid H^{-1}(B) \in \mathbb{F}\right\} .
\end{aligned}
$$

This is easily seen to be a $t$-structure, so, by Theorem 3.6. $B:=\mathbb{D} \leq 0 \cap \mathbb{D} \geq 0$ is an abelian category. More precisely, $B$ is equivalent to the full subcategory of $\mathcal{D}(A)$ consisting of complexes $d: A^{-1} \rightarrow A^{0}$ such that ker $d \in \mathbb{F}$ and coker $d \in \mathbb{T}$.

Remark 4.2. Let $\mathbb{T}^{\prime}$ and $\mathbb{F}^{\prime}$ be essential images of $\mathbb{F}[1]$ and $\mathbb{T}$ in $\mathrm{B}$. Then $\left(\mathbb{T}^{\prime}, \mathbb{F}^{\prime}\right)$ is a torsion theory for B. (However, if we repeat the same process as above for this torsion theory, we may not get back A.) 


\section{Appendix I: TOPOLOGICAL TRIANGUlAted CATEGORIES}

References: ([Ho], Section 7), [Ma], (We, Section 10.9).

In our examples, we considered only triangulated categories that come from algebra (e.g, derived categories). There are certain triangulated categories that arise from topology (e.g, stable homotopy categories) that we have not discussed in these notes but which are worthy of attention. In line with our topological-vs-chaincomplex comparison picture of Lecture 2, we say a few words about the homotopy category of spectra.

Spectra. Recall from Lecture 2 that the cone construction (which is the main input in the construction of derived categories) was motivated by a topological construction on spaces. One may wonder then whether one could repeat the construction of the derived categories in the topological setting. The problem that arises here is that the suspension functor $X \mapsto S X$ is not an auto-equivalence of the category of topological spaces (while its chain complex counterpart, the shift functor $C \mapsto C[1]$, is). To remedy this, one can formally "invert" the suspension functor. This gives rise to the category of Spanier-Whitehead spectra ([Ma], Chapter 1) from which we can construct a triangulated category by inverting weak equivalences, in the same way we obtained the derived category by inverting quasi-isomorphisms.

What made topologists unhappy about the category of Spanier-Whitehead spectra is that it is not closed under arbitrary colimits. 3 Nowadays there are better categories of spectra that do not have this deficiency. They go under various names such as spectra, $\Omega$-spectra, symmetric spectra, etc. All these categories come equipped with a notion of weak equivalence, and inverting the weak equivalences in any of these categories gives rise to the same triangulated category, the homotopy category of spectra. Understanding the homotopy category of spectra is an area of active research that is called stable homotopy theory. To see how triangulated categories emerge in this context consult the given references.

\section{Appendix II: Different Illustrations of TR4}

One way of illustrating TR4 is via the following "braid" diagram:

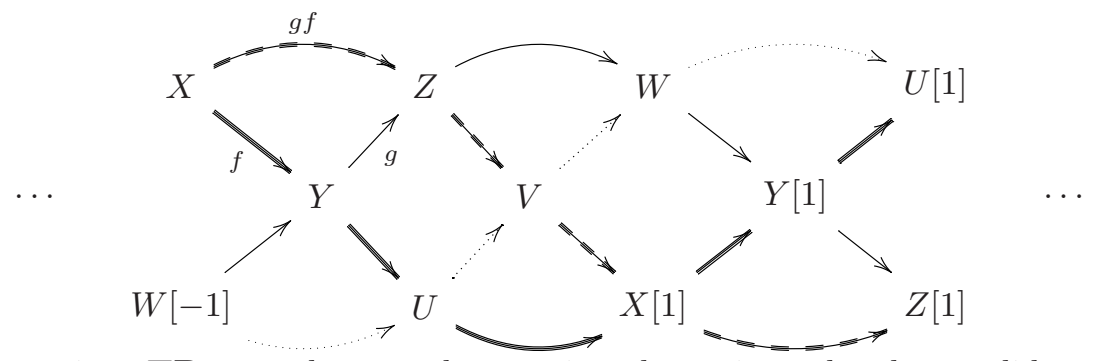

The axiom TR4 can be stated as saying that, given the three solid strands of braids, the dotted strand can be filled so that the diagram commutes. It is understood that each of the four long sequences is exact (i.e., obtained from a distinguished triangle). Note that we are requiring the existence of only three

\footnotetext{
${ }^{3}$ Actually neither are the bounded derived categories!
} 
consecutive dotted arrows; the rest of the sequence is uniquely determined by these three.

Another depiction of the axiom TR4 is via the following octahedron:

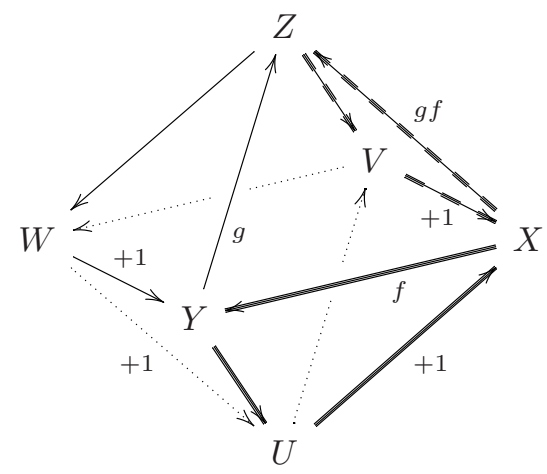

The axiom requires that the dotted arrows could be filled. It is understood that the four mixed-color triangles are commutative, and the four unicolor triangles are exact.

\section{REFERENCES}

[Ad] J. F. Adams, Stable homotopy and generalised homology, Chicago Lectures in Mathematics. University of Chicago Press, Chicago, IL, 1995.

[BeBeDe] A. A. Beilinson, J. Bernstein, P. Deligne, Faisceaux pervers, Analysis and topology on singular spaces, I (Luminy, 1981), 5-171, Astérisque, 100, Soc. Math. France, Paris, 1982

[BoKa] A. I. Bondal, M. M. Kapranov, Framed triangulated categories, (Russian) Mat. Sb. 181 (1990), no. 5, 669-683; translation in Math. USSR-Sb. 70 (1991), no. 1, 93-107.

[Fr] P. Freyd, Abelian categories, Harper, 1966.

[Ga] P. Gabriel, Des catégories abéliennes, Bull. Soc. Math. France 90 (1962), 323-448.

[GeMa] S. I. Gelfand, Y. I. Manin, Methods of homological algebra, Springer-Verlag, Berlin, 1996.

[Gr] A. Grothendieck, Sur quelques points d'algébre homolgiques, Tôhoku Math. J. 9 (1957), $119-221$.

[HaReSm] D. Happel, I. Reiten, S. O. Smalø, Tilting in abelian categories and quasitilted algebras, Mem. Amer. Math. Soc. 575.

[Ha1] R. Hartshorne, Algebraic geometry, Graduate Texts in Mathematics 52, Springer-Verlag, New York-Heidelberg, 1977.

[Ha2] R. Hartshorne, Residues and duality, Lecture Notes in Math. 20, Springer-Verlag, 1966.

[HiSt] P. Hilton, U. Stammbach, A course in homological algebra, Graduate Texts in Mathematics 4, Springer-Verlag, New York, 1997.

[Ho] M. Hovey, Model categories, Mathematical Surveys and Monographs 63, American Mathematical Society, Providence, RI, 1999.

[Iv] B. Iversen, Cohomology of sheaves, Universitext, Springer-Verlag, Berlin, 1986.

[Bernhard Keller's Webpage] http://www.math.jussieu.fr/\%7Ekeller/publ/index.html

[Ke1] B. Keller, Introduction to abelian and derived categories, in Representations of reductive groups, edited by R. W. Carter and M. Geck, Cambridge University Press 1998, 41-62 (available on Keller's webpage).

[Ke2] B. Keller, Derived categories and their uses, in Handbook of algebra, edited by M. Hazewinkel, Elsevier 1996 (available on Keller's webpage).

[Ke3] B. Keller, On differential graded categories, preprint (available on Keller's webpage).

[Ke4] B. Keller, Introduction to $A_{\infty}$-algebras and modules, Homology, Homotopy, and Applications 3 (2001), 1-35 (available on Keller's webpage).

[KeVo] B. Keller, D. Vossieck, Aisles in derived categories, Bull. Soc. Math. Belg. Ser. A 40 (1988), no. 2, 239-253.

[KaSch] M. Kashiwara, P. Schapira, Sheaves on Manifolds, Grundlehren der Mathematichen Wissenschaften 292, Springer-Verlag, 1990. 
[Li] J. Lipman, Notes on derived categories and derived functors, available at http://www.math.purdue.edu/ lipman.

[Ma] S. Mac Lane, Categories for the working mathematician, Second edition. Graduate Texts in Mathematics 5, Springer-Verlag, New York, 1998.

[Ma] H. R. Margolis, Spectra and the Steenrod algebra North-Holland Mathematical Library 29, North-Holland Publishing Co., Amsterdam, 1983.

[May1] J. P. May A concise course in algebraic topology, Chicago Lectures in Mathematics, University of Chicago Press, Chicago, IL, 1999.

[May2] J. P. May, The axioms for triangulated categories, preprint.

[Mi1] B. Mitchel, The full embedding theorem, Am. J. Math. 86 (1964), 619-637.

[Mi2] B. Mitchel, Rings with several objects, Adv. in Math. 8 (1972), 1-161.

[Ne] A. Neeman, Triangulated categories, Annals of Mathematics Studies, 148. Princeton University Press, Princeton, NJ, 2001.

[Po] A. Polishchuk, Noncommutative two-tori with real multiplication as noncommutative projective varieties, J. Geom. Phys. 50, no. 1-4, 162-187.

$[\mathrm{Pu}]$ D. Puppe, On the formal structure of stable homotopy theory, in Colloq. Alg. Topology, Aarhus University (1962), 65-71.

[Ro] A. Rosenberg, The spectrum of abelian categories and reconstructions of schemes, in Rings, Hopf Algebras, and Brauer groups, 257-274, Lectures Notes in Pure and Appl. Math. 197, Marcel Dekker, New York, 1998.

[Ve] J.-L. Verdier, Des catégories dérivées des catégories abéliennes, Astérisque 239, 1996.

[We] C. A. Weibel, Introduction to homological algebra, Cambridge Studies in Advanced Mathematics 38, Cambridge University Press, 1994.

E-mail address: behrang@alum.mit.edu

Mathematics Department, Florida State University, 208 Love Building, Tallahassee, FL 32306-4510, U.S.A. 